\title{
ANAPLASMA, EHRLICHIA AND RICKETTSIA SPECIES INFECTIONS IN CATS European guidelines from the ABCD on prevention and management
}

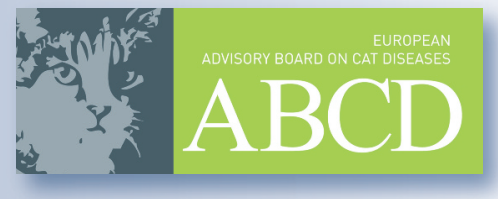

\author{
Maria Grazia Pennisi, Regina Hofmann-Lehmann, Alan D Radford, \\ Séverine Tasker, Sándor Belák, Diane D Addie, Corine Boucraut-Baralon, \\ Herman Egberink, Tadeusz Frymus, Tim Gruffydd-Jones, Katrin Hartmann, \\ Marian C Horzinek*, Margaret J Hosie, Albert Lloret, Hans Lutz, \\ Fulvio Marsilio, Etienne Thiry, Uwe Truyen and Karin Möstl
}

Overview: Anaplasma species, Ehrlichia species and Rickettsia species are vector-borne pathogens infecting a wide variety of mammals, but causing disease in very few of them.

Infection in cats: Anaplasma phagocytophilum is the most important feline pathogen among these rickettsial organisms, and coinfections are possible. Little information is available on the pathogenesis of these agents in cats. Clinical signs are usually reported soon after tick infestation. They are mostly non-specific, consisting of fever, anorexia and lethargy. Joint pain may occur. Infection in humans: Some rickettsial species (A phagocytophilum, Ehrlichia chaffeensis, Ehrlichia ewingii, Rickettsia conorii, Rickettsia rickettsii, Rickettsia felis, Rickettsia typhi and Candidatus Neoehrlichia mikurensis) are of zoonotic concern. Direct contact with cat saliva should be avoided because of potential contamination by $R$ felis. Infected cats are 'sentinels' of the presence of rickettsial pathogens in ticks and fleas in a given geographical area, and they signal a risk for people exposed to vectors.

*Deceased - see 'Acknowledgements' on page 547.

\section{Agent properties and epidemiology}

Obligate intracellular gram-negative coccoid organisms of the Anaplasma, Ehrlichia and Rickettsia genera are vector-borne members of the Rickettsiales order, infecting humans and a wide variety of domestic and wild animals worldwide. ${ }^{1}$ They generally have a wide host specificity, evidenced by the fact that many mammalian species can be infected. Importantly, some hosts might serve as reservoirs of infection; however, susceptibility to disease is usually more restricted.

Anaplasma, Ehrlichia and Rickettsia species infections have been generally less studied in cats (Table 1) than in dogs. These organisms are difficult to culture in vitro. The advent of molecular genetics has, however, opened new avenues for the study of their infection biology. ${ }^{1}$

\section{Anaplasmataceae family}

Anaplasma and Ehrlichia species are tick-borne pathogens of the Anaplasmataceae family and are pleomorphic intravacuolar organisms that replicate in haemopoietic cells. They give rise to a variety of cytoplasmic inclusions - small elementary bodies (0.2-0.4 $\mu \mathrm{m}$ diameter), larger reticulate bodies and morulae (up to 2-6 $\mu \mathrm{m}$ ).

Anaplasma phagocytophilum replicates in myeloid cells (mostly in neutrophils; Figure 1) and is the agent of granulocytotropic anaplasmosis. It infects people and a wide range of animal species worldwide, especially in the northern hemisphere, and is the most important feline pathogen of the Anaplasma genus. Wild small mammals are the natural reservoirs. Antibody prevalence to A phagocytophilum in cats has been reported as 4.5-33.3\% in Italy, $2.0-8.0 \%$ in Spain, ${ }^{5,6} 16.2 \%$ in Germany, ${ }^{7} 22.1 \%$ in Sweden, ${ }^{8}$ and $1.8-38.0 \%$ in the USA. ${ }^{9-11}$ A phagocytophilum DNA was recently amplified in $0.4 \%$ of blood samples from cats admitted to veterinary clinics in southern Germany. ${ }^{12}$

Anaplasma platys replicates in mature platelets and is the agent of thrombocytotropic anaplasmosis, a disease well documented worldwide in dogs. ${ }^{13}$ It has occasionally been detected in cats by PCR analysis. ${ }^{11,14,15}$

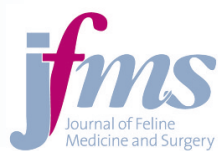

European Advisory Board on Cat Diseases

www.abcdcatsvets.org

Corresponding author: Maria Grazia Pennisi

Email: mariagrazia.pennisi@unime.it 


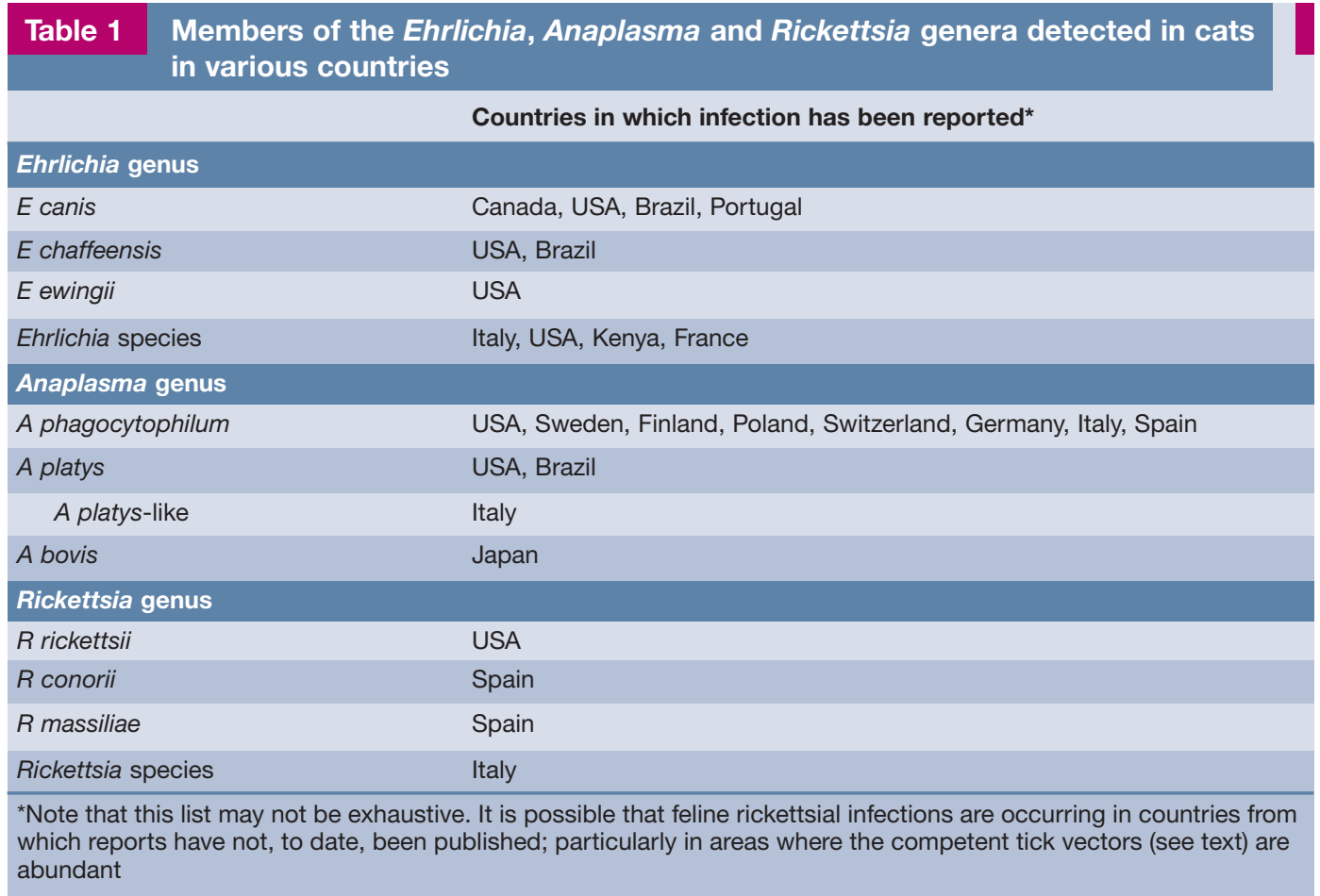

European Advisory
Board on Cat Diseases
The European Advisory
Board on Cat Diseases
(ABCD) is a body of
experts in immunology,
vaccinology and clinical
feline medicine that issues
guidelines on prevention
and management of feline
infectious diseases in
Europe, for the benefit of
the health and welfare
of cats. The guidelines
are based on current
scientific knowledge of
the diseases and available
vaccines concerned.
Any future update of the
Anaplasma, Ehrlichia
and Rickettsia species
infections in cats
guidelines will be
available at
www.abcdcatsvets.org

Anaplasma bovis has also been found by PCR, in feline blood samples from Japan. ${ }^{16}$ Recently a novel, unclassified $A$ platys-like strain from cats was characterised in Sardinia (Italy). This strain, despite its tropism for platelets, is closely related to others identified in ruminants. ${ }^{17}$

Ehrlichia canis is the agent of canine monocytotropic ehrlichiosis, a disease described in tropical and temperate regions worldwide, with the exception of Australia. Ehrlichia chaffeensis is the agent of human monocytotropic ehrlichiosis, which has been reported mainly in the USA. The granulocytotropic pathogen Ehrlichia ewingii has been found in dogs and humans in midwestern and southern regions of the USA. In cats, $E$ canis has been detected by PCR in blood samples. ${ }^{6,11,18-24}$ Less frequently, E chaffeensis and $E$ ewingii have also been detected. ${ }^{11,20}$ Cats seropositive for $E$ canis have been reported in Europe $(6.0-18.0 \%),{ }^{3-6,25,26}$ Brazil $(5.5 \%)^{20}$ and the USA $(0.7 \%){ }^{11}$

Another member of the Anaplasmataceae family is 'Candidatus Neoehrlichia mikurensis' (genus 'Candidatus Neoehrlichia'), which leads to neoehrlichiosis, an emerging zoonosis. This tick-borne agent has been found mainly in immunocompromised patients, and in ticks and rodents, and recently also in two dogs, 27,28 although so far not in cats. The infection may be underdiagnosed because diagnostic assays are not yet widely available.
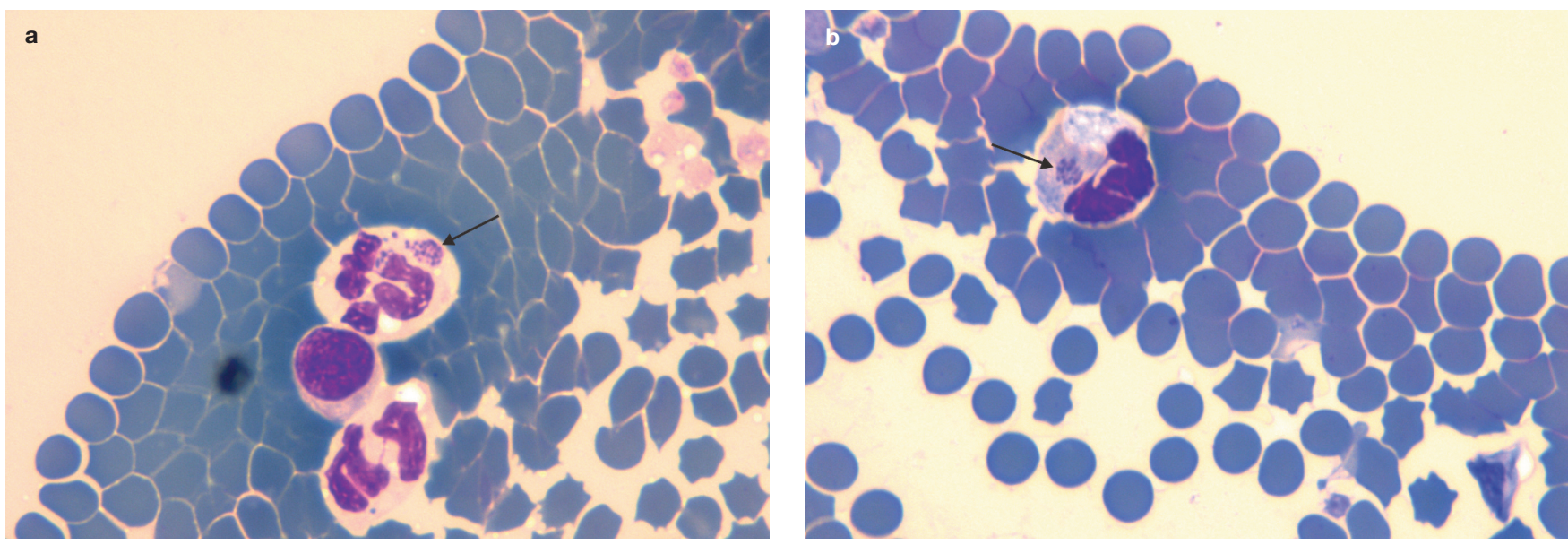

Figure 1 Presence of Anaplasma species morulae (arrows) in neutrophils from a 2-year-old male European shorthair cat (a) and a 6-year-old female European shorthair cat (b) in Poland. Reprinted with permission from Adaszek et al² 


\section{Rickettsiaceae family}

The Rickettsiaceae family includes the spotted fever group (SFG) and the typhus group agents. $^{1}$

There are more than 20 species within the SFG of the Rickettsia genus, some of which are important human pathogens. Historically, the most important zoonotic agents have been Rickettsia conorii (the cause of Mediterranean spotted fever in the Old World) and Rickettsia rickettsii (the agent of Rocky Mountain spotted fever in the Americas). However, recent molecular studies have increasingly focused on other rickettsial species that may be involved in human clinical disease. Rickettsia massiliae, for example, is now recognised as the most widely distributed rickettsial species that affects humans, being found worldwide. ${ }^{29}$

$R$ rickettsii and $R$ conorii are both transmitted by ticks and infect dogs, sometimes causing clinical disease. Less information is available about the effect of these agents in cats. Feline infections involving $R$ massiliae and $R$ conorii have been confirmed (both by PCR and antibody testing) in endemic areas of Spain and Portugal, 5,30-32 and cats seropositive for $R$ rickettsii have been reported in the USA. ${ }^{33}$

Rickettsia felis is emerging worldwide as a flea-borne SFG human pathogen; it is detected frequently in Ctenocephalides felis, less often in other flea species. ${ }^{29} \mathrm{C}$ felis is the vector and the recognised reservoir of $R$ felis, which is vertically transmitted to successive generations of fleas. ${ }^{34}$ Recent studies in parts of Europe have shown that Ctenocephalides infection rates range from $2.8 \%$ (in Albania) ${ }^{35}$ to $54.2 \%$ (in Andalucia, Spain). ${ }^{36}$ Cats are susceptible to $R$ felis infection and seroconvert after exposure to infected fleas. However, they are probably not reservoir hosts as they experience short-term bacteraemia (blood PCR test results are usually negative in antibodypositive cats). ${ }^{4,32,37,38}$ In rare cases, $R$ felis DNA can be amplified also from the skin or gingiva of cats with negative blood PCR results. ${ }^{39}$ It is unknown whether $R$ felis is present in other tissues of seropositive cats and whether it should be considered a feline pathogen.

Rickettsia typhi is a worldwide flea-borne rickettsial pathogen of the typhus group. It is the agent of murine or endemic typhus, transmitted to humans, cats or wild animal reservoirs by infected rat (Xenopsylla species) or cat (C felis) fleas.

Just as in humans and dogs, coinfections with multiple vector-borne pathogens occur in cats. The consequences of such mixed infections are unknown. ${ }^{11,15,23,40}$

\section{Geographical distribution}

The geographical distribution of Anaplasma, Ehrlichia and Rickettsia pathogens overlaps with that of their competent vectors. In Europe, two tick species are principally involved: Rhipicephalus sanguineus sensu lato (the brown dog tick or kennel tick), which is the main tick vector of $E$ canis and $R$ conorii (and suspected for $A$ platys); and Ixodes ricinus (the black-legged tick), the vector of $A$ phagocytophilum. Both species are found on cats. ${ }^{41-43}$

I ricinus has a wide distribution, extending from the Mediterranean area to Scandinavian countries, and from Portugal to Ukraine. ${ }^{44}$ In Eastern Europe a closely related species, Ixodes persulcatus, is found..$^{13} R$ sanguineus sensu lato is common in the Mediterranean basin; in northern countries, $R$ sanguineus is not indigenous but it can hibernate sheltered in cracks of kennel structures, with the result that its area of distribution is expanding northwards.

In summary, $E$ canis and $A$ platys are considered endemic in Mediterranean countries but are spreading northward, whereas $A$ phagocytophilum is reported mainly in Northern and Central Europe. ${ }^{13}$ In stray cats in northern Italy, blood PCR for A phagocytophilum, Ehrlichia species and Rickettsia species tested positive with a prevalence of $17.7 \%, 5.4 \%$ and $31.9 \%$, respectively. ${ }^{45}$ Similar rates were found in healthy cats and cats showing clinical signs. Blood transfusion consequently may be a nonvectorial mode of transmission for rickettsial agents in cats, as is well recognised in dogs. ${ }^{13}$

\section{Blood}

transfusion

may be a

non-vectorial

mode of

transmission

for rickettsial

agents in cats,

as is well

recognised

in dogs.

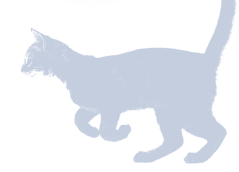

\section{Pathogenesis and clinical signs}

Little information is available on the pathogenesis of rickettsial agents in cats.

A limited number of studies involving experimental infections or exposure to A phagocy tophilum or $R$ felis in cats exist. In one such study, intraperitoneal inoculation of A phagocytophilum-infected blood in a small number of cats resulted in mild disease, with only transient fever and no associated changes in appetite or general appearance. However, mild reductions in total leukocyte, neutrophil and lymphocyte counts, significant reductions in packed cell volume, and a transient increase in alanine transaminase and aspartate aminotransferase values were detected. $^{46}$ Antinuclear antibodies and increased expression of IFN $\gamma$ mRNA were also found in infected cats, but they showed normal antibody responses to feline herpesvirus and feline leukaemia virus vaccination 2 weeks postinfection. When experimental infection with A phagocytophilum was performed in feline immunodeficiency virusinfected cats, upregulation of IL-10 expression was observed instead of IFN $\gamma$, but the clinical course of anaplasmosis was similar. ${ }^{46}$

Recent experimental exposure of four cats to wild-caught adult Ixodes scapularis ticks induced a largely subclinical dual infection with A phagocytophilum and Borrelia burgdorferi. ${ }^{40}$ In fact, a transient lymphopenia was the only abnormality detected during monitoring of the cats' general appearance, appetite, body 
temperature and complete blood count for 13 weeks after tick infestation. ${ }^{40}$

In naturally exposed cats, clinical signs of feline granulocytotropic anaplasmosis are usually reported soon after tick infestation. They are mostly non-specific, consisting of fever, anorexia, lethargy and dehydration. Clinicopathological abnormalities include mild or moderate thrombocytopenia, anaemia and lymphopenia. ${ }^{2,47-51}$ Lameness and swollen joints, epistaxis or pain on abdominal palpation have been less frequently reported. ${ }^{2,47,52}$ The clinical course is usually short and not severe; abnormalities resolve quickly, particularly when antibiotic treatment is given.

Natural infection with $A$ platys in a cat in Brazil was associated with anorexia, lethargy, urinary tract infection and thrombocytopenia. ${ }^{14}$ In another cat, persistent $A$ platys infection was found concurrently with Bartonella henselae, Bartonella koehlerae and 'Candidatus Mycoplasma haemominutum' infections; the cat was also diagnosed with multiple myeloma. ${ }^{15}$ In both cases, the pathogenic role of $A$ platys was not clearly established. In the cat with multiple myeloma, the associated immune system dysfunction could have been responsible for increased susceptibility to the coinfections. ${ }^{53}$

Experimental subcutaneous inoculation of cats with a canine strain of E canis was not successful in inducing infection, and the pathogenesis of feline monocytotropic ehrlichiosis in cats is not known. ${ }^{54}$ Natural disease has been confirmed by molecular testing in only a few cases and mainly manifests as nonspecific signs such as fever, anorexia and lethargy; more rarely, hyperaesthesia, joint pain, pale mucous membranes, lymph node and spleen enlargement, and haemorrhagic diathesis (petechiae, vitreous haemorrhage) have also been reported. ${ }^{54}$ Haematology can reveal non-regenerative anaemia, thrombocytopenia, pancytopenia and increased or decreased white cell counts. ${ }^{54}$ Bone marrow hypoplasia is found in cats with pancytopenia or anaemia and thrombocytopenia on haematology. ${ }^{18}$ The most consistent biochemical abnormality seen with feline monocytotropic ehrlichiosis has been hyperproteinaemia and polyclonal or monoclonal gammopathy, which is also typical of the chronic course of canine monocytotropic ehrlichiosis. ${ }^{54}$ Antinuclear antibodies have been found in some cats and neutrophilic polyarthritis was diagnosed in a cat with joint signs. ${ }^{18}$

\section{Clinical signs of rickettsial infection in cats}

are mostly non-specific, including fever, anorexia, lethargy and dehydration.

In a study evaluating clinically ill cats for evidence of rickettsial infection, no association was found between antibody positivity and fever, and no febrile cat was found to be PCR positive for $R$ felis or $R$ rickettsii. ${ }^{55}$ Another study investigated possible links between Ehrlichia species or A phagocytophilum infections and anaemia, but no significant associations were detected. ${ }^{56}$

\section{Diagnosis}

The main indication for undertaking diagnostic investigations for rickettsial disease is a febrile syndrome affecting cats exposed to ticks in endemic areas; in particular, strays or outdoor pet cats not protected by the regular use of appropriate ectoparasiticides.

Blood or buffy-coat smear evaluation may be useful for cytological diagnosis of Ehrlichia and Anaplasma species infections. In general, intracytoplasmic inclusion bodies are more frequently found in granulocytotropic anaplasmosis than in monocytotropic ehrlichiosis. A phagocytophilum inclusion bodies are found in 1-24\% of circulating neutrophils in cats with natural granulocytotropic anaplasmosis. In experimentally infected cats they appear 7-9 days postinfection, ${ }^{46}$ or over the first 10 weeks after tick infestation. ${ }^{40}$ Following antibiotic therapy they are no longer visible. ${ }^{40,47,50}$ With A platys, bacteraemia is cyclical, recurring at 1-2 week intervals, with a higher percentage of circulating infected platelets during the early cycles. ${ }^{57}$

Antibodies to rickettsial infections can be detected by immunofluorescence and ELISA techniques. Cross-reaction is possible between A phagocytophilum and A platys but not E canis, although $E$ canis can cross-react with other Ehrlichia species. Antibodies against A phagocytophilum were detected in an experimental study within 14 days of infection, and seroconversion occurs in natural infections even in antibiotic-treated cats. ${ }^{46}$ In cats experimentally exposed to infected ticks, antibodies against A phagocytophilum appeared 2-6 weeks after infestation. ${ }^{40}$ In the case of a positive

The main indication for diagnostic investigations for rickettsial disease is a febrile syndrome affecting cats exposed to ticks in endemic areas; in particular, strays or outdoor pet cats not protected by ectoparasiticides. 


\section{Recommendations to avoid zoonotic transmission}

Rickettsial pathogens are transmitted to humans by competent vectors. Infected cats, as well as dogs, are 'sentinels' of the presence of rickettsial pathogens in ticks and fleas in a given geographical area and they signal a risk for people exposed to vectors.
Regular application of ectoparasiticides to pets reduces the risk of exposure of humans to vectors of rickettsial agents. Direct contact with cat saliva should be avoided because of the potential for contamination by $R$ felis, as well as other zoonotic pathogens. immunofluorescence test at first sampling, a four-fold increase in the titre over 4 weeks may confirm the acute course of the infection. ${ }^{46-48,50}$ Some cats testing positive to E canis by PCR were found to be antibody negative despite the chronic course of their disease. ${ }^{18}$ This suggests that a negative antibody test does not rule out a diagnosis of ehrlichiosis in cats with compatible clinical signs.

Blood PCR analysis is a sensitive and specific method for confirming diagnosis at the onset of acute clinical signs when antibody testing is usually still negative. ${ }^{40,46}$ Because of overlapping clinical signs, the use of genusinclusive primers for Ehrlichia-Anaplasma and Rickettsia species genera in PCRs has been suggested, followed by sequencing of any resulting PCR products to determine the infecting species. ${ }^{1}$ However, a recent study has demonstrated that some genus-specific PCRs also detect Pseudomonas sequences and may lead to false-positive results that may only be recognised after sequencing analysis. ${ }^{28}$ Alternatively, species-specific real-time TaqMan assays may be faster and more sensitive options for the molecular detection of rickettsaemia.

Samples for microscopic detection or PCR should be collected prior to the initiation of antibiotic treatment.

\section{Treatment}

Doxycycline is the antibiotic of choice for treating rickettsial infections in cats, although currently there are only retrospective case reports supporting this recommendation [EBM grade

\section{EBM grades}

The ranking system for grading the level of evidence of the statement within the treatment section is described on page 574 of the July 2015 Special Issue (see page 548 for more information). III]. ${ }^{2,47,48,50,51}$ It is administered at 10 $\mathrm{mg} / \mathrm{kg}$ PO q24h for 28 days. Clinical improvement is seen in the first $24-48 \mathrm{~h}$, unless coinfections not susceptible to doxycycline (eg, protozoal vector-borne agents) are present, or other complications develop such as severe bleeding.
Animals generally respond well to treatment but may remain persistently infected.

In cases testing negative by microscopy, PCR or serology, or while awaiting results and where there is a strong clinical suspicion of rickettsial infection - treatment should be initiated to prevent the potential rapid progression of clinical disease.

respond well

to doxycycline

treatment but

may remain

persistently infected.

\section{Cats generally Prevention}

As vectors are the main routes of transmission of rickettsial infections, regular application of appropriate ectoparasiticide spot-on treatments or collars may protect cats from becoming infected, as is well recognised in dogs.

In endemic areas, cats should be tested for rickettsial blood-borne infections to confirm they are negative before being used as blood donors. ${ }^{58}$

\section{KaY POINTS}

* Anaplasma species, Ehrlichia species and Rickettsia species are vector-borne pathogens, the most important of which in cats is Anaplasma phagocytophilum.

\$ The geographical distribution of these rickettsial organisms overlaps with that of their competent vectors (ticks or fleas).

* Blood or buffy-coat smear evaluation may be useful for cytological diagnosis of Ehrlichia and Anaplasma species infections.

* Antibodies to rickettsial infections can be detected by immunofluorescence and ELISA techniques, but cross-reactions may occur between organisms of the same genus.

* Blood PCR analysis is a sensitive and specific method for confirming diagnosis at the onset of acute clinical signs and before starting therapy, when antibody testing is usually still negative.

Doxycycline is the first-choice antibiotic for treating rickettsial infections.

* Regular application of appropriate ectoparasiticide spot-on treatments or collars protects cats from infection.

* In endemic areas, blood donors should be tested for rickettsial blood-borne infections. 


\section{Acknowledgements}

The ABCD is deeply indebted to Marian C Horzinek, the 'spiritus rector' and past chairman of $\mathrm{ABCD}$ for many years, who sadly passed away during the finalisation of these guidelines.

\section{Conflict of interest}

The authors do not have any potential conflicts of interest to declare.

\section{Funding}

The authors received no specific grant from any funding agency in the public, commercial or not-for-profit sectors for the preparation of this article. The ABCD is supported by Merial, but is a scientifically independent body and its members receive no stipends from Merial.

\section{References}

1 Allison RW and Little SE. Diagnosis of rickettsial diseases in dogs and cats. Vet Clin Pathol 2013; 42: 127-144.

2 Adaszek L, Górna M, Skrzypczak M, et al. Three clinical cases of Anaplasma phagocytophilum infection in cats in Poland. J Feline Med Surg 2013; 15: 333-337.

3 Ebani VV and Bertelloni F. Serological evidence of exposure to Ehrlichia canis and Anaplasma phagocytophilum in Central Italian healthy domestic cats. Ticks Tick Borne Dis 2014; 5: 668-671.

4 Persichetti MF, Solano-Gallego L, Serrano L, et al. Detection of vector-borne pathogens in cats and their ectoparasites in southern Italy. Parasit Vectors 2016; 9: 247.

5 Solano-Gallego L, Hegarty B, Espada Y, et al. Serological and molecular evidence of exposure to arthropod-borne organisms in cats from northeastern Spain. Vet Microbiol 2006; 118: 274-277.

6 Ayllón T, Diniz PP, Breitschwerdt EB, et al. Vectorborne diseases in client-owned and stray cats from Madrid, Spain. Vector Borne Zoonotic Dis 2012; 12: 143-149.

7 Hamel D, Bondarenko A, Silaghi C, et al. Seroprevalence and bacteremia of Anaplasma phagocytophilum in cats from Bavaria and Lower Saxony (Germany). Berl Munch Tierarztl Wochenschr 2012; 125: 163-167.

8 Elfving K, Malmsten J, Dalin AM, et al. Serological and molecular prevalence of Rickettsia helvetica and Anaplasma phagocytophilum in wild cervids and domestic mammals in the central parts of Sweden. Vector Borne Zoonotic Dis 2015; 15: 529-534.

9 Magnarelli LA, Bushmich SL, Ijdo JW, et al. Seroprevalence of antibodies against Borrelia burgdorferi and Anaplasma phagocytophilum in cats. Am J Vet Res 2005; 66: 1895-1899.

10 Billeter SA, Spencer JA, Griffin B, et al. Prevalence of Anaplasma phagocytophilum in domestic felines in the United States. Vet Parasitol 2007; 147: 194-198.

11 Hegarty BC, Qurollo BA, Thomas B, et al. Serological and molecular analysis of feline vector-borne anaplasmosis and ehrlichiosis using species-specific peptides and PCR. Parasit Vectors 2015; 8: 320.
12 Bergmann M, Englert T, Stuetzer B, et al. Prevalence of selected rickettsial infections in cats in Southern Germany. Comp Immunol Microbiol Infect Dis 2015; 42: 33-36.

13 Sainz A, Roura X, Miró G, et al. Guideline for veterinary practitioners on canine ehrlichiosis and anaplasmosis in Europe. Parasit Vectors 2015; 8: 75.

14 Lima MLF, Soares PT, Ramos CAN, et al. Molecular detection of Anaplasma platys in a naturally-infected cat in Brazil. Braz J Microbiol 2010; 41: 381-385.

15 Qurollo BA, Balakrishnan N, Cannon CZ, et al. Co-infection with Anaplasma platys, Bartonella henselae, Bartonella koehlerae and 'Candidatus Mycoplasma haemominutum' in a cat diagnosed with splenic plasmacytosis and multiple myeloma. J Feline Med Surg 2014; 16: 713-720.

16 Sasaki H, Ichikawa Y, Sakata Y, et al. Molecular survey of Rickettsia, Ehrlichia, and Anaplasma infection of domestic cats in Japan. Ticks Tick Borne Dis 2012; 3: 307-310.

17 Zobba R, Anfossi AG, Visco S, et al. Cell tropism and molecular epidemiology of Anaplasma platys-like strains in cats. Ticks Tick Borne Dis 2015; 6: 272-280.

18 Breitschwerdt EB, Abrams-Ogg AC, Lappin MR, et al. Molecular evidence supporting Ehrlichia canis-like infection in cats. J Vet Intern Med 2002; 16: 642-649.

19 Oliveira LS, Mourão LC, Oliveira KA, et al. Molecular detection of Ehrlichia canis in cats in Brazil. Clin Microbiol Infect 2009; 15: 53-54.

20 Braga M, André MR, Freschi CR, et al. Molecular and serological detection of Ehrlichia spp. in cats on São Luís island, Maranhão, Brazil. Rev Bras Parasitol Vet 2012; 21: 37-41.

21 Braga IA, Santos LG, Melo AL, et al. Hematological values associated to the serological and molecular diagnostic in cats suspected of Ehrlichia canis infection. Rev Bras Parasitol Vet 2013; 22: 470-474.

22 Braga IA, Santos LG, Ramos DG, et al. Detection of Ehrlichia canis in domestic cats in the centralwestern region of Brazil. Braz J Microbiol 2014; 45: 641-645.

23 Maia C, Ramos C, Coimbra M, et al. Bacterial and protozoal agents of feline vector-borne diseases in domestic and stray cats from southern Portugal. Parasit Vectors 2014; 7: 115.

24 André MR, Herrera HM, Fernandes Sde J, et al. Tick-borne agents in domesticated and stray cats from the city of Campo Grande, state of Mato Grosso do Sul, Midwestern Brazil. Ticks Tick Borne Dis 2015; 6: 779-786.

25 Aguirre E, Tesouro MA, Amusatequi I, et al. Assessment of feline ehrlichiosis in central Spain using serology and a polymerase chain reaction technique. Ann N Y Acad Sci 2004; 1026: 103-105.

26 Ortuño A, Gauss CB, García F, et al. Serological evidence of Ehrlichia spp. exposure in cats from northeastern Spain. J Vet B Infect Dis Vet Public Health 2005; 52: 246-248.

27 Diniz PP, Schulz BS, Hartmann K, et al. “Candidatus Neoehrlichia mikurensis" infection in a dog from Germany. J Clin Microbiol 2011; 49: 2059-2062. 
28 Hofmann-Lehmann R, Wagmann N, Meli ML, et al. Detection of 'Candidatus Neoehrlichia mikurensis' and other Anaplasmataceae and Rickettsiaceae in Canidae in Switzerland and Mediterranean countries. Schweiz Arch Tierheilkd 2016; 158: 691-700.

29 Brouqui P, Parola P, Fournier PE, et al. Spotted fever rickettsioses in southern and eastern Europe. FEMS Immunol Med Microbiol 2007; 49: 2-12.

30 Alves AS, Milhano N, Santos-Silva M, et al. Evidence of Bartonella spp., Rickettsia spp. and Anaplasma phagocytophilum in domestic, shelter and stray cat blood and fleas, Portugal. Clin Microbiol Infect 2009; 15: 1-3.

31 Vilhena H, Martinez-Díaz V, Cardoso L, et al. Feline vector-borne pathogens in the north and centre of Portugal. Parasit Vectors 2013; 6: 99.

32 Segura F, Pons I, Miret J, et al. The role of cats in the eco-epidemiology of spotted fever group diseases. Parasit Vectors 2014; 7: 353.

33 Case JB, Chomel B, Nicholson W, et al. Serological survey of vector-borne zoonotic pathogens in pet cats and cats from animal shelters and feral colonies. J Feline Med Surg 2006; 8: 111-117.

34 Wedincamp J, Jr and Foil LD. Vertical transmission of Rickettsia felis in the cat flea (Ctenocephalides felis Bouché). J Vector Ecol 2002; 27: 96-101.

35 Silaghi C, Knaus M, Rapti D, et al. Survey of Toxoplasma gondii and Neospora caninum, haemotropic mycoplasmas and other arthropodborne pathogens in cats from Albania. Parasit Vectors 2012; 7: 62.

36 Márquez FJ, Muniain MA, Rodríquez-Liebana JJ, et al. Incidence and distribution pattern of Rickettsia felis in peridomestic fleas from Andalusia, Southeast Spain. Ann N Y Acad Sci 2006; 1078: 344-346.

37 Wedincamp J, Jr and Foil LD. Infection and seroconversion of cats exposed to cat fleas (Ctenocephalides felis Bouché) infected with Rickettsia felis. J Vector Ecol 2000; 25: 123-126.

38 Hawley JR, Shaw SE and Lappin MR. Prevalence of Rickettsia felis DNA in the blood of cats and their fleas in the United States. J Feline Med Surg 2007; 9: 258-262.

39 Lappin MR and Hawley J. Presence of Bartonella species and Rickettsia species DNA in the blood, oral cavity, skin and claw beds of cats in the United States. Vet Dermatol 2009; 20: 509-514.

40 Lappin MR, Chandrashekar R, Stillman B, et al. Evidence of Anaplasma phagocytophilum and Borrelia burgdorferi infection in cats after exposure to wild-caught adult Ixodes scapularis. J Vet Diagn Invest 2015; 27: 522-525.

41 Jameson LJ and Medlock JM. Tick surveillance in Great Britain. Vector Borne Zoonotic Dis 2011; 11: 403-412.

42 Claerebout E, Losson B, Cochez C, et al. Ticks and associated pathogens collected from dogs and cats in Belgium. Parasit Vectors 2013; 6: 183.

43 Pennisi MG, Persichetti MF, Serrano L, et al. Ticks and associated pathogens collected from cats in Sicily and Calabria (Italy). Parasit Vectors 2015; 8: 512.

44 ECDC. Tick species - distribution maps. European Centre for Disease Prevention and Control. http: / / ecdc.europa.eu/en/healthtopics/vectors / vector-maps / Pages / VBORNET-maps-tickspecies.aspx (accessed October 1, 2106).

45 Spada E, Proverbio D, Galluzzo P, et al. Molecular study on selected vector-borne infections in urban stray colony cats in northern Italy. J Feline Med Surg 2014; 16: 684-688.

46 Foley JE, Leutenegger CM, Dumler JS, et al. Evidence for modulated immune response to Anaplasma phagocytophila sensu lato in cats with FIV-induced immunosuppression. Comp Immunol Microbiol Infect Dis 2003; 26: 103-113.

47 Bjöersdorff A, Svendenius L, Owens JH, et al. Feline granulocytic ehrlichiosis - a report of a new clinical entity and characterization of the infectious agent. I Small Anim Pract 1999; 40: 20-24.

48 Lappin MR, Breitschwerdt EB, Jensen WA, et al. Molecular and serological evidence of Anaplasma phagocytophilum infection in cats in North America. J Am Vet Med Assoc 2004; 225: 893-896.

49 Schaarschmidt-Kiener D, Graf F, von Loewenich FD, et al. Anaplasma phagocytophilum infection in a cat in Switzerland. Schweiz Arch Tierheilkd 2009; 151: 336-341.

50 Heikkilä HM, Bondarenko A, Mihalkov A, et al. Anaplasma phagocytophilum infection in a domestic cat in Finland: case report. Acta Vet Scand 2010; 52: 62.

51 Savidge C, Ewing P, Andrews J, et al. Anaplasma phagocytophilum infection of domestic cats: 16 cases from northeastern USA. J Feline Med Surg 2016; 18: 85-91.

52 Tarello W. Microscopic and clinical evidence for Anaplasma (Ehrlichia) phagocytophilum infection in Italian cats. Vet Rec 2005; 156: 772-774.

Three Special Issues of JFMS containing ABCD guidelines on feline infectious disease - some well recognised, others lesser known - have been published:

Infectious diseases, Prevention and Management: Volume 11, July 2009

- Infectious diseases, Part 2: Volume 15, July 2013

* Infectious diseases, Part 3: Volume 17, July 2015

Available at:

cpsi.jfms.com
53 Pratt G, Goodyear $O$ and Moss P. Immunodeficiency and immunotherapy in multiple myeloma. Br J Haematol 2007; 138: 563-579.

54 Lappin MR and Breitschwerdt ER. Ehrlichia spp. infection (feline monocytotropic ehrlichiosis). In Greene CE (ed). Infectious diseases of the dog and cat. St Louis, MO: Elsevier, 2012, pp 238-241.

55 Bayliss D, Morris AK, Horta MC, et al. Prevalence of Rickettsia species antibodies and Rickettsia species DNA in the blood of cats with and without fever. J Feline Med Surg 2009; 11: 266-270.

56 Ishak AM, Radecki S and Lappin MR. Prevalence of Mycoplasma haemofelis, "Candidatus Mycoplasma haemominutum", Bartonella species, Ehrlichia species, and Anaplasma phagocytophilum DNA in the blood of cats with anemia. J Feline Med Surg 2006; 9: 1-7.

57 Harvey JW, Simpson CF and Gaskin JM. Cyclic thrombocytopenia induced by a Rickettsia-like agent in dogs. J Infect Dis 1978; 137: 182-188.

58 Pennisi MG, Hartmann K, Addie DD, et al. Blood transfusion in cats. ABCD guidelines for minimising risks of infectious iatrogenic complications. J Feline Med Surg 2015; 17: 589-593. 